\title{
Latency Reversal 2.0: Giving the Immune System a Seat at the Table
}

\author{
Vidisha Singh $^{1} \cdot$ Amir Dashti $^{1} \cdot$ Maud Mavigner ${ }^{1,2} \cdot$ Ann Chahroudi $^{1,2,3}$ (B)
}

Accepted: 21 December 2020 / Published online: 12 January 2021

(C) The Author(s) 2021

\begin{abstract}
Purpose of Review For most people living with HIV (PLWH), treatment with effective antiretroviral therapy (ART) results in suppression of viremia below the limit of detection of clinical assays, immune reconstitution, reduced immune activation, avoidance of opportunistic infections, and progression to AIDS. However, ART alone is not curative, and HIV persists in a non-replicating, latent form. In this review, we provide a historical perspective on non-specific latency reversal approaches (LRA 1.0) and summarize recent advances in latency reversal strategies that target specific signaling pathways within CD4+ $\mathrm{T}$ cells or other immune cells to induce expression of latent HIV (immune-based latency reversal, or LRA 2.0).

Recent Findings The HIV reservoir is primarily composed of latently infected CD4+ T cells carrying integrated, replicationcompetent provirus that can give rise to rebound viremia if ART is stopped. Myeloid lineage cells also contribute to HIV latency in certain tissues; we focus here on CD4+ T cells as a sufficient body of evidence regarding latency reversal in myeloid cells is lacking. The immunomodulatory LRA 2.0 approaches we describe include pattern recognition receptor agonists, immune checkpoint inhibitors, non-canonical NF-kB stimulation, and transient CD8+ lymphocyte depletion, along with promising combination strategies. We highlight recent studies demonstrating robust latency reversal in nonhuman primate models.

Summary While significant strides have been made in terms of virus reactivation from latency, initial hopes for latency reversal alone to result in a reduction of infected cells, through viral cytopathic effect or an unboosted immune system, have not been realized and it seems clear that even effective latency reversal strategies will need to be paired with an approach that facilitates immune recognition and clearance of cells containing reactivated virus.
\end{abstract}

Keywords HIV-1 cure $\cdot$ Immune-based therapeutics $\cdot$ Shock and kill $\cdot$ Latency reversal agents $\cdot$ SMAC $\cdot$ CD8 depletion

\section{Brief Historical Perspective on LRA 1.0}

Following the initial descriptions of HIV persistence despite prolonged suppression of viremia in individuals on suppressive ART, approaches to target the latent reservoir by activating T cells were proposed. Clinical trials in the late 1990searly 2000s used IL-2 alone or in combination with anti-CD3

This article belongs to the Topical Collection on HIV Pathogenesis and Treatment

Ann Chahroudi

ann.m.chahroudi@emory.edu

1 Department of Pediatrics, Emory University School of Medicine, Atlanta, GA, USA

2 Center for Childhood Infections and Vaccines of Children's Healthcare of Atlanta and Emory University, Atlanta, GA, USA

3 Yerkes National Primate Research Center, Emory University Atlanta, Atlanta, GA, USA antibodies to reverse HIV latency. This approach of global T cell activation had to be halted due to severe toxicity leading notably to acute renal failure, seizures, and hypothyroidism and was followed by more targeted strategies aimed at inducing HIV gene expression [1-4].

The next latency reversal agents (LRA) developed were designed to target HIV epigenetic silencing, a major regulator of viral latency that includes DNA methylation and histone post-translational modifications such as histone acetylation. The latency reversal activity of several histone deacetylase inhibitors (HDACi) including romidepsin, panobinostat, vorinostat, and valproic acid have been tested in clinical trials. Despite initial hopes, clinical studies with valproic acid did not demonstrate an impact on viral transcription $[5,6]$. However, in 2012, it was reported that a single dose of vorinostat administered to 7 participants on suppressive ART induced a significant increase in cell-associated unspliced (CA US) HIV-1 RNA in resting $\mathrm{CD}^{+} \mathrm{T}$ cells [7]. Similar results were observed in follow-up studies during 
vorinostat multiple-dose therapy $[8,9]$. It has to be noted that an increase in viremia, which is arguably the best read out for latency reversal (and to which we henceforth refer to as "onART viremia"; see Table 1 for assays used to quantify latency reversal), was not observed in any of these studies. In this regard, panobinostat treatment resulted in a qualitative increase in on-ART viremia compared to baseline in a subset of study participants [24]. Romidepsin infusions in 6 individuals on long-term ART also led to transient increases in plasma viral loads and CA US HIV-1 RNA levels in total $\mathrm{CD} 4^{+} \mathrm{T}$ cells [25]. The size of the viral reservoir was unchanged in each of these HDACi trials. Other epigenetic modifiers more recently explored as potential LRA include DNA and histone methyltransferase inhibitors (HMTi) targeting transcription initiation similarly to HDACi, as well as bromodomain and extra terminal (BET) inhibitors that promote transcription elongation. Notably, the HMTi's chaetocin and BIX-01294 have been shown to increase HIV-1 recovery from ex vivo cultures of resting $\mathrm{CD}^{+} \mathrm{T}$ cells and small-molecule inhibitor of BET bromodomains such as JQ1, RVX-208, and PFI-1 appears to activate HIV transcription in latently infected Jurkat $T$ cells [26-28]. These approaches have not yet been tested in PLWH. Collectively, the clinical studies to date show that LRA 1.0 approaches resulted in modest increases in CA US HIV-1 RNA, rare and limited increases in viremia, and no reduction of the viral reservoir in study participants maintained on suppressive ART.

\section{Immunomodulatory LRA $\mathbf{2 . 0}$}

New classes of molecules with immunomodulatory properties are being explored as potential LRA including pattern recognition receptor (PRR) ligands and immune checkpoint inhibitors (ICIs). These approaches have the theoretical advantage of simultaneously reactivating HIV and restoring immune functions to facilitate elimination of the infected cells. For the purpose of this review, we will focus on the latency reversal activity of these agents (Fig. 1).

\section{PRR Agonists}

Pattern recognition receptors (PRR) are central players of innate immunity involved in pathogen sensing. Unlike agents that target epigenetic silencing, PRR agonists indirectly activate $\mathrm{CD}^{+} \mathrm{T}$ cells and HIV transcription presumably through stimulation of antigen presenting cells followed by proinflammatory cytokine release, although precise mechanisms have not been delineated for every agonist. Several toll-like receptor (TLR) agonists have reached pre-clinical and clinical testing of their ability to reverse HIV latency. The most extensively studied have been TLR-7 agonists that trigger type I interferon production by plasmacytoid dendritic cells (pDCs) with downstream activation of CD4+ T cells. In 2018, it was reported that two TLR-7 agonists (GS-986 and GS-9620) induced in vivo viral reactivation as well as reservoir reduction in the rhesus macaque (RM) model of simian immunodeficiency virus (SIV) infection and suppressive ART [29•]. Viral reactivation was demonstrated by transient increases in viremia while ART was maintained. Furthermore, 2/9 RM treated with GS-9620 did not show viral rebound after ART interruption and adoptive transfer of peripheral blood mononuclear cells (PBMCs) and lymph node mononuclear cells from these two RM to two uninfected animals did not result in establishment of infection. However, these exciting results showing virus reactivation were not reproduced in multiple additional studies in RM infected with SIV or simian human immunodeficiency virus (SHIV) $[30 \bullet, 31 \bullet, 32 \cdot, 33,34]$ nor in PLWH. Vesatolimod (formerly GS-9620) was assessed in a phase $1 \mathrm{~b}$, randomized, double-blind, placebo-controlled clinical trial, and only isolated viral load elevations above 20 copies $/ \mathrm{ml}$ (highest 69 copies $/ \mathrm{ml}$ ) were observed [35].

The TLR-9 ligand MGN1703 that also stimulates type I interferon from $\mathrm{pDCs}$ was also advanced to clinical trials. A single-arm study reported quantifiable on-ART viremia (211571 copies $/ \mathrm{ml}$ ) in 6/15 HIV-1-infected individuals, during a short-course treatment with MGN1703 (also called lefitolimod) [36]. However, a subsequent study failed to demonstrate a virological benefit of MGN1703 administration with unchanged US CA HIV RNA levels and a stable reservoir as evaluated by total HIV DNA or replication-competent virus levels in $\mathrm{CD}^{+} \mathrm{T}$ cells and time to rebound following ATI [37]. An effect on on-ART viremia was not reported in this study and in a similar trial of a different TLR-9 agonist (CpG-ODN7909) [38], although presumably if significant increases were seen, these would have been included in the published results. The safety and efficacy of MGN1703 will be further assessed in combination with broadly neutralizing antibodies in a randomized clinical trial (NCT03837756). The TLR-3 agonist Poly-ICLC has also been evaluated but did not induce significant latency reversal [39]. Overall, the TLR agonists tested thus far have not proven to be reproducibly successful as LRA and most have the disadvantage of not directly acting upon $\mathrm{CD} 4+\mathrm{T}$ cells.

In addition to the well-characterized TLR, cytosolic PRR have been explored as potential targets of the shock approach including retinoic acid-inducible gene-I (RIG-I)-like receptors (RLRs), and stimulator of interferon genes (STING). Acitretin, an FDA-approved retinoic acid derivative that enhances RIG-I signaling, has been shown to increase HIV transcription in vitro and induce preferential apoptosis of HIVinfected cells [40]. The STING pathway activates interferon regulatory factors and the nuclear factor kappa $\mathrm{B}(\mathrm{NF}-\mathrm{kB})$ and could thus act indirectly through antigen presenting cells and directly on T cells. The STING ligands 2'3'-cGAMP and c-dAMP have been reported to increase SIV RNA levels and 


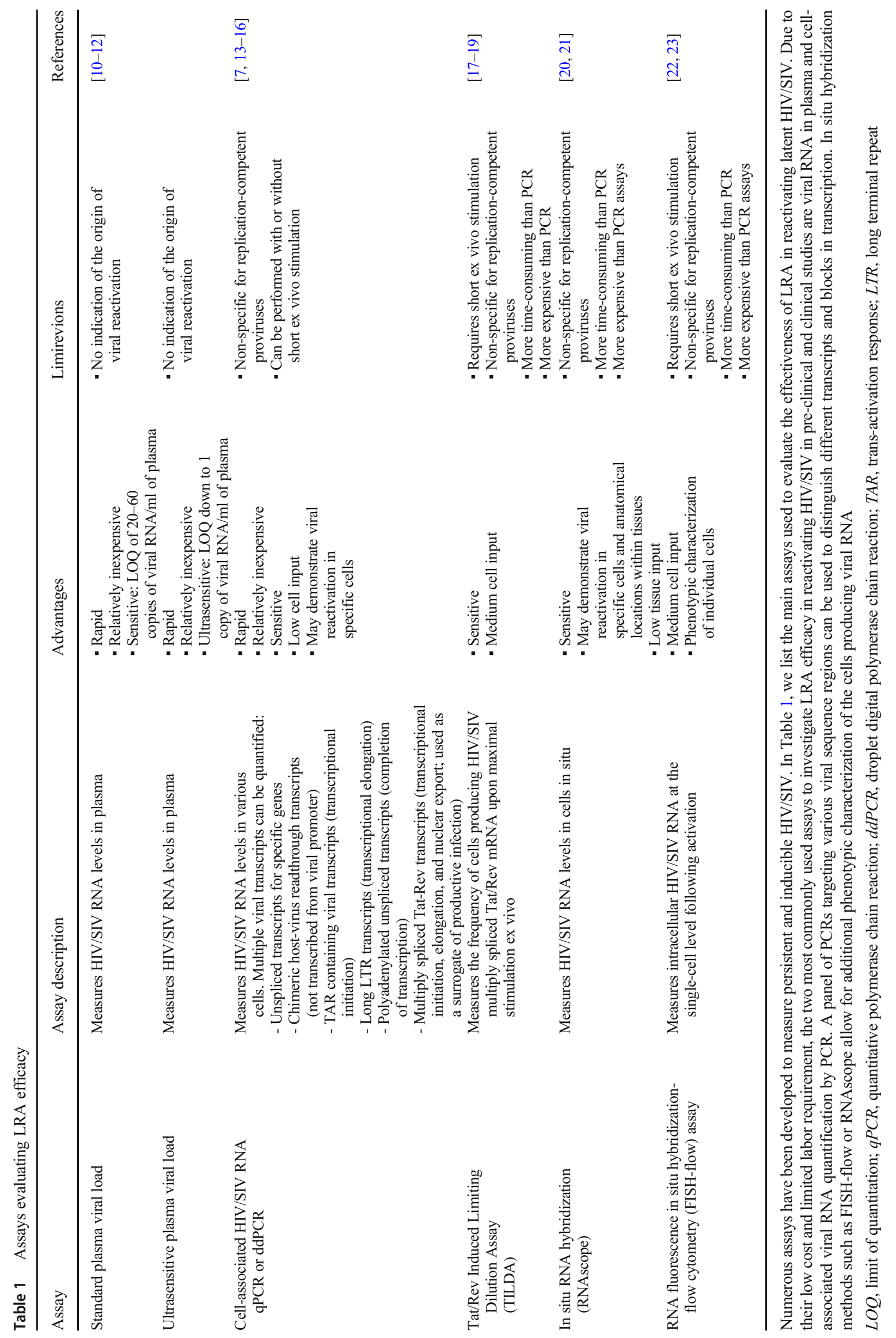




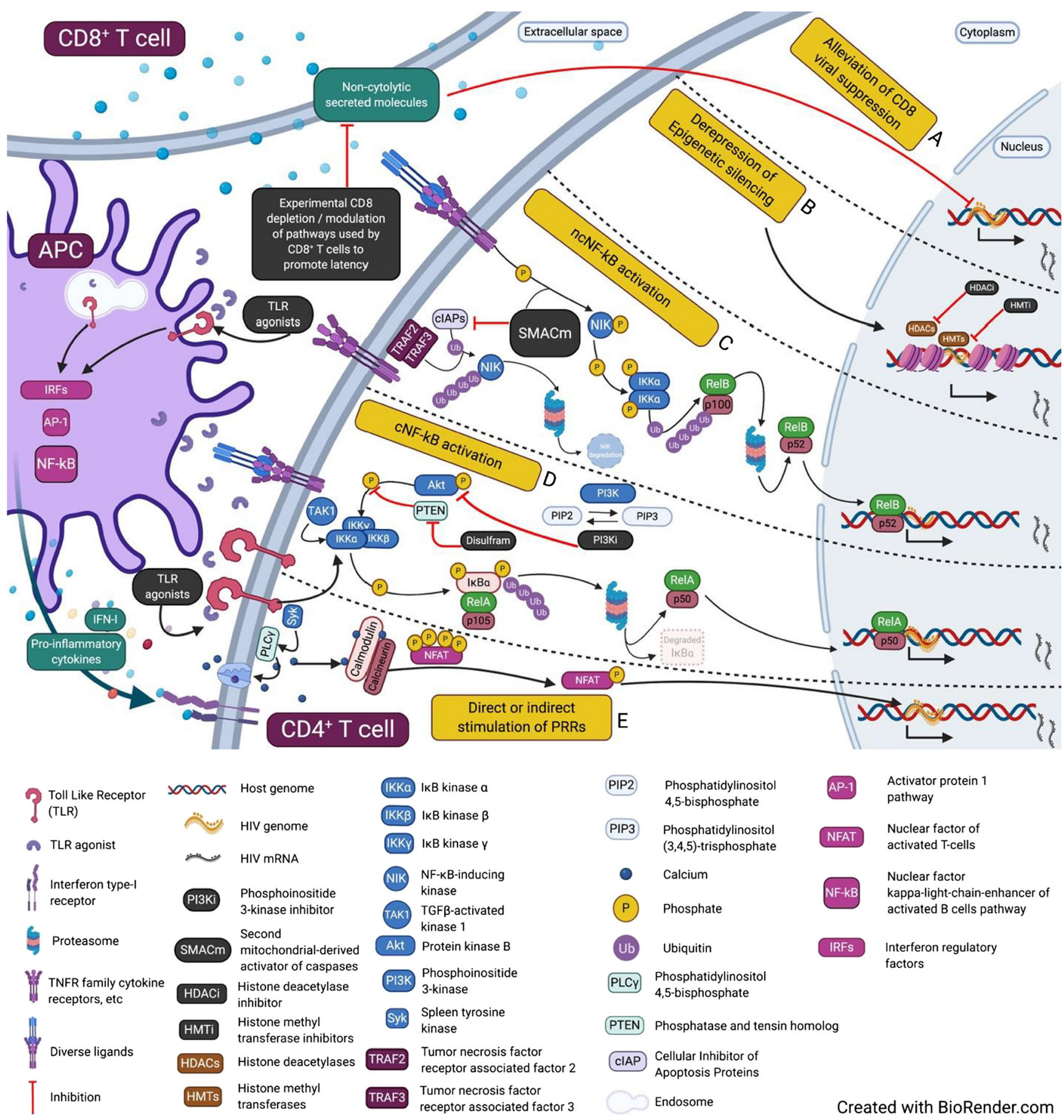

decrease SIV DNA levels ex vivo in PBMCs isolated from cynomolgus macaques with natural control of viremia [41]. The combination of cGAMP and the HDACi resminostat was also shown to induce a significant increase in HIV reactivation and apoptosis in HIV-infected cells in vitro [42]. Ongoing work in our laboratory and that of M. Paiardini is exploring the virological and immunological impacts of STING agonist administration in vivo in RM during the acute phase of SIV infection or after sustained suppression of viremia on ART.

\section{Immune Checkpoint Inhibitors}

The dysregulation of the immune system observed during chronic HIV infection involves a progressive exhaustion of $\mathrm{CD}^{+} \mathrm{T}$ cells characterized by the overexpression of coinhibitory receptors, such as PD-1 (programmed cell death1), CTLA-4 (cytotoxic T lymphocyte-associated protein 4), LAG-3 (lymphocyte-activation gene 3), TIGIT (T cell immunoreceptor with immunoglobulin and ITIM 
Fig. 1 Latency reversal agents and their known or proposed mechanisms of action. (A) Alleviation of CD8 viral suppression: non-cytolytic molecules secreted by CD8+ T cells suppress HIV RNA production by mechanisms yet to be fully elucidated. Experimental CD8 depletion alleviates CD8+ $\mathrm{T}$ cell suppression leading to viral reactivation. Identifying the specific molecular pathways used by CD8+ T cells to promote latency might be a key to maximal latency reversal. (B) Derepression of epigenetic silencing: HDACi's and HMTi's unwind the genome thereby increasing access of transcription factors to targeted genes for expression. (C) Non-canonical NF- $\kappa$ B pathway activation: in a normal and unstimulated condition, NIK is regulated by the action of TRAF3 ligase binding to NIK and TRAF2 binding to cIAPs. This results in constant ubiquitination and degradation of NIK, ultimately maintaining low-levels of NIK. Under cellular stimulation by diverse ligands, receptor binding leads to TRAF3 degradation and consequent NIK accumulation, thereby enabling nuclear entry of the transcription factors of RelB and $\mathrm{p} 52$. This pathway can also be activated by binding of SMACm to cIAPs, resulting in NIK accumulation and similar downstream events. (D) Canonical NF-KB pathway activation: stimulation of a surface receptor leads to recruitment of TGF $\beta$-activated kinase 1 (TAK1), activation of the IKK complex, and ultimately release of the transcription factors RelA and $\mathrm{p} 50$ to translocate to the nucleus. PI3K inhibitors prevent Akt activation thereby enabling nuclear entry of NF- $\kappa$ B. (E) Direct or indirect stimulation of PRR: stimulation of antigen presenting cells (APCs) via surface or endosomal TLR) activates the canonical NF- $\mathrm{kB}$, AP-1, or IRF pathways resulting in secretion of pro-inflammatory cytokines (including type I interferons) which indirectly leads to latency reversal in CD4+ T cells. Stimulation of TLR on CD4+ T cells can also directly activate the canonical NF-kB or NFAT pathways. Similar activities of other PRR agonists have been described

domains), or Tim-3 (T cell immunoglobulin and mucin domain-containing protein 3 ). In addition, co-inhibitory receptors are thought to contribute to HIV latency and are preferentially expressed at the surface of latently HIVinfected CD4+ T cells [43-48]. As such, blockade of coinhibitory receptors represents a valuable therapeutic approach that could both restore immune functions of the exhausted HIV-specific T cells and reverse latency. As ICIs were initially developed for cancer therapy, several reports of their impact on HIV persistence come from observations of PLWH with coexisting malignancies. Increases in CA US HIV RNA in CD4+ T cells were observed following treatment with the anti-CTLA-4 antibody ipilimumab in an individual with metastatic melanoma, and interestingly, cyclic decreases in viremia using a single copy assay were also noted (rather than the increased onART viremia we would expect to see with effective latency reversal) [49]. This same individual received a single intravenous infusion of anti-PD-1 nivolumab resulting in a significant increase in CA US HIV RNA but again no significant change in plasma HIV RNA levels [44]. However, the impact of immune checkpoint inhibitors on latency reversal is inconsistent in these case reports [50-52]. Interestingly, a pre-clinical study in ART-suppressed SIV-infected RM treated with monoclonal antibodies targeting PD-1 and/or CTLA-4 suggested viral reactivation as demonstrated by on-ART viremia in a fraction of the treated animals as well as a reduction of the reservoir as shown by a significantly decreased level of cell-associated SIV DNA in effector memory $\mathrm{CD}^{+}{ }^{+} \mathrm{T}$ cells and a decreased frequency of intact provirus [53・*]. As autoimmune-related side effects have now been seen in multiple clinical trials of ICIs $[54,55]$, the future of this approach to reverse latency is uncertain.

\section{Non-canonical NF-kB Stimulation as LRA $\mathbf{2 . 0}$}

\section{The NF-кB Pathway (Canonical vs Non-Canonical)}

Recent work from our group and others has highlighted the promise of selective activation of the non-canonical NF- $\mathrm{KB}$ pathway for HIV and SIV latency reversal in $\mathrm{CD} 4+\mathrm{T}$ cells. The NF-KB family includes 5 inducible transcription factors: NF-kB1 (p50), NF-kB2 (p52), RELA (p65), RELB, and cREL. Activation of the classical or canonical NF- $k B$ pathway, mediated by cell surface stimulation, recruitment of adaptor molecules to convert the IKK complex (inhibitor of nuclear factor $\mathrm{kB}$ ), and a series of phosphorylation, ubiquitination, and finally degradation steps for IKB proteins, mostly triggers the transcription factors NF-KB1, RELA, and c-REL [56]. The canonical NF- $K B$ pathway activates a diverse and broad range of genes and the response to stimuli is rapid and transient [57]. A second mode of NF- $\mathrm{KB}$ pathway activation, termed noncanonical, selectively and predominantly activates NF- $\mathrm{kB} 2$ and RELB in a strictly inducible manner through processing of $\mathrm{p} 100$. Response to stimuli in the non-canonical NF-KB (ncNF- $\mathrm{kB}$ ) pathway is slow but persistent and transcription occurs in a more narrow set of genes compared to the canonical NF- $k B$ pathway [57]. A central component of the $n c N F-\kappa B$ pathway is NIK (NF- $\kappa \mathrm{B}$-inducing kinase) through which all pathway inducers are known to signal [58]. In the unstimulated condition, newly synthesized NIK is constantly ubiquitinated and degraded by TRAF3 [59]. Specifically, TRAF3 recruits TRAF2 that binds to cellular inhibitor of apoptosis 1 and 2 (cIAP1, cIAP2). Cell surface binding of ligands to TNFRs (BAFFR, CD40, CD30, CD27, etc.) stimulates TRAF3 degradation, NIK accumulation, IKKa activation, and p100 phosphorylation and degradation. In addition to receptor ligation, the ncNF-kB pathway can be activated by intermediates of the apoptosis cascade such as the second mitochondrial activator of caspases (SMAC) that induce the degradation of cIAP and thus NIK activation. Peptide mimetics with SMAC-like activity, termed SMAC mimetics (SMACm), were developed initially to promote apoptosis in tumor cells and have now been shown to induce HIV and SIV latency reversal.

Protein kinase $\mathrm{C}$ agonists (PKCa) that activate NF- $\mathrm{KB}$ through the canonical pathway have been explored as LRA. 
However, due to toxicity concerns related to the potent and broad activation of signaling pathways, only one clinical trial has been performed to date. Administration of two different single doses of bryostatin-1 failed to induce HIV-1 RNA transcription as evaluated by US CA RNA levels in PBMCs and on-ART viremia in a pilot double-blind phase I clinical trial [60]. An alternative to PKCa is represented by disulfiram, a drug used to treat chronic alcoholism, that activates the phosphoinositide-3-kinase (PI3K)/Akt pathway that interacts with the NF- $\mathrm{KB}$ cascade resulting in nuclear entry of NF-KB1 and RELA (canonical transcription factors). Two clinical trials of short-term administration of disulfiram showed no to limited effect on HIV transcription with only a twofold increase in CA US HIV-1 RNA at the highest dose tested [61, 62]. In both studies, the viral reservoir size was not reduced following treatment with disulfiram.

\section{SMAC Mimetics}

In 2015, Pache and colleagues showed that several SMACm induced increased levels of HIV transcription in latently infected Jurkat cells by stimulating the ncNF- $\mathrm{kB}$ pathway [63*0]. A synergistic effect on latency reversal activity was also seen when combining SMACm with panobinostat in vitro. Similar results were observed with the SMACm Debio 1143 in a subsequent study showing latency reversal in vitro and ex vivo in resting $\mathrm{CD}^{+} \mathrm{T}$ cells isolated from ART-suppressed PLWH and humanized bone marrow/liver/ thymic (BLT) mice [64].

Our group recently demonstrated that activation of the ncNF- $\mathrm{KB}$ signaling pathway by the SMACm AZD5582 reversed both HIV and SIV latency in vivo as shown by the induction of viral RNA expression in the blood and tissues of ARTsuppressed HIV-infected BLT humanized mice and SIVinfected RM. ART-suppressed HIV-1JR-CSF-infected BLT mice were given a single injection of SMACm leading to onART viremia in $>50 \%$ of mice. Additionally, comparison of HIV RNA level in resting $\mathrm{CD}^{+} \mathrm{T}$ cells isolated from various tissues of ART-suppressed BLT mice including the bone marrow, thymic organoid, lymph node, spleen, liver, and lungs showed increased levels in SMACm-treated animals vs controls. Furthermore, we also evaluated AZD5582 latency reversal activity in twelve RM infected by $\mathrm{SIV}_{\text {mac239 }}$ and treated with a potent ART regimen for over a year before receiving weekly infusions of SMACm for 3 or 10 weeks while ART was maintained. Increased expression of several mediators of the ncNF- $\mathrm{kB}$ pathway was confirmed by RNA sequencing, including NF- $\mathrm{KB} 2$ and RELB. On-ART viremia was observed in RM treated with AZD5582 (5/12 RM using a standard viral load assay and 8/12 RM using an ultrasensitive assay). The highest measurement was $\sim 10^{3}$ copies $/ \mathrm{ml}$ with multiple instances of sustained viremia between AZD5582 infusions. In the RM who received ten doses of AZD5582, cell-associated SIV RNA was also significantly higher in resting $\mathrm{CD} 4^{+} \mathrm{T}$ cells isolated from lymph nodes as compared to controls and the replication-competent reservoir in lymph node CD4+ T cells was reduced. No further impact of AZD5582 treatment on the viral reservoir size was observed [65*0]. In unpublished work, we have found that treatment of SIV-infected ART-suppressed RM with a second cycle of the SMACm AZD5582 can induce on-ART viremia $>60$ copies $/ \mathrm{ml}$ in $75 \%$ of RM that were initially not responsive. More recently, Pache et al. replicated the finding of latency reversal in vivo using a humanized mouse model with the SMACm Ciapavir [66].

Although a robust decline in the CD4+ $\mathrm{T}$ cell reservoir was not observed in these studies, SMACm have been suggested to selectively target and kill HIV-infected resting memory CD4+ T cells and macrophages by an autophagy-dependent mechanism $[67,68]$. Besides the potential for targeting apoptosis of infected cells, SMACm present the major advantage of being more specific than most LRA developed so far, thus limiting safety concerns. Collectively, these results suggest that activating the ncNF- $\mathrm{kB}$ pathway using SMACm is an efficient strategy to reactivate HIV/SIV that warrants further investigation.

\section{Transient CD8+ Lymphocyte Depletion as LRA 2.0}

While it is known that CD8+ T cells utilize cytolytic mechanisms of viral control during HIV infection, a non-cytolytic role for $\mathrm{CD} 8+\mathrm{T}$ cells in promoting viral latency has also been described. Several in vitro and in vivo studies have demonstrated that, both in the presence and absence of ART, CD8+ T cells exert a suppressive effect on virus production in HIV/ SIV-infected CD4+ T cells. In early evidence from in vitro experiments, removal of $\mathrm{CD} 8+\mathrm{T}$ cells from HIV-infected PBMCs consistently yielded high levels of HIV reverse transcriptase (RT) activity in supernatant in contrast with undepleted PBMC cultures from the same donors that was unrelated to an effect on cell killing [69]. A dose-dependent relationship between level of viral suppression (measured by reverse transcriptase activity) and number of CD8+ T cells with controlled reintroduction of autologous $\mathrm{CD} 8+\mathrm{T}$ cells was found. A substantial portion of the more recent evidence for $\mathrm{CD} 8+$ cells promoting viral latency comes from studies in nonhuman primates in which experimental depletion of CD8+ lymphocytes can be accomplished by monoclonal antibody administration. The two commonly used antibodies for this purpose are MT-807R1 which targets the CD8 $\alpha$ chain and is highly effective in transiently depleting CD8+ T and NK cells, and $\mathrm{CD} 8 \mathrm{~b} 255 \mathrm{R} 1$ which targets the $\mathrm{CD} 8 \beta$ chain and selectively removes $\mathrm{CD} 8+\mathrm{T}$ cells, albeit to a lesser extent and duration than MT-807R1 [70]. Below, we summarize the experimental data supporting this non-cytolytic role and the utility of such findings for latency reversal agents in curative research. 
Studies of untreated SIV infection in which CD8a depletion was performed with MT-807R1 have demonstrated the efficacy of $\mathrm{CD} 8+\mathrm{T}$ cells in controlling viral replication, and this research was extended to incorporate continuous ART administration after SIV/SHIV infection to model the latent state in vivo. In a cohort of SIV-infected RM, it was found that CD8+ $\mathrm{T}$ cells were required for maintaining viral suppression during short-term ART [71]. In 100\% of animals undergoing CD8+ lymphocyte depletion with MT-807R1, plasma viral load increases were detected during the depletion period ( $>$ $90 \%$ depletion of circulating CD8+ T cells), and subsequent $\mathrm{CD} 8+\mathrm{T}$ cell reconstitution correlated with reversion to viral control.

Following this initial paradigm shifting experiment, three independent in vivo studies from our group and that of $\mathrm{G}$. Silvestri demonstrated enhanced latency reversal when CD8+ lymphocyte depletion was combined with another agent. Given substantial heterogeneity within the latently infected pool, combination LRA treatments are hypothesized to achieve more potent and broader virus reactivation. In the first study, a single administration of MT-807R1 was given prior to four weekly doses of the IL-15 superagonist (N-803) in SIV-infected, long-term ART-suppressed macaques [72••]. N-803 was selected based upon its latency reversal activity in vitro [73]. All macaques showed virus production in plasma post-CD8+ lymphocyte depletion and N-803 administration, some to levels as high as $10^{4}$ copies $/ \mathrm{ml}$, and on-ART viremia continued for several weeks until CD8+ T cells were reconstituted $[72 \bullet \bullet]$. Findings were replicated in HIVinfected humanized mice and in an in vitro latency and reversal assay (LARA) that uses autologous CD8+ T cells isolated prior to HIV infection, indicating that the suppressive effect of CD8+ $\mathrm{T}$ cells is not restricted to antigen-experienced, virusspecific $\mathrm{T}$ cells. In each of these experiments (SIV-infected macaques, HIV-infected humanized mice, and HIV-infected human PBMCs), the combination of CD8 depletion and $\mathrm{N}-803$ showed significantly greater virus reactivation compared to CD8 depletion or N-803 administration alone; however, viral rebound dynamics were not altered in comparison to controls when ART was interrupted. As a proof-of-concept, we also demonstrated that suboptimal depletion of CD8+ T cells using the CD8b255R1 antibody given with four weekly doses of N-803 in ART-suppressed, SHIV-infected macaques induced virus reactivation in three out of five macaques [70]. Finally, the CD8a-depleting antibody was given in tandem with the SMACm AZD5582 that we have shown to be a highly effective LRA when used alone. The combination of CD8+ lymphocyte depletion and five weekly doses of the SMACm AZD5582 in SIV-infected ART-suppressed macaques resulted in on-ART viremia in $100 \%$ of treated animals [74]. As described earlier, in a similar cohort of ARTsuppressed SIV-infected macaques, the effect of AZD5582 treatment alone was less extensive, with only $56 \%$ of animals experiencing viremia $>60$ copies/ml [65••].

As it may not be practical to implement CD8 depletion studies in HIV-infected individuals, nailing down the precise mechanism(s) through which these cells are repressing virus production may aid in the identification of more targeted therapies that block this effect while CD8+ T cells remain.

A recent in vitro study aimed to better characterize the nature of the observed CD8+ T cell effect on latency and identified a non-MHC-dependent, non-cytolytic capacity of CD8+ T cells to suppress HIV replication through silencing of LTR-dependent viral transcription [75]. Co-culture of CD4+ T cells infected in vitro with the controlled addition of autologous (non-HIV exposed) $\mathrm{CD} 8+\mathrm{T}$ cells resulted in increased levels of integrated HIV DNA, reduced CD4+ T cell activation, increased CD4+ T cell survival, and promotion of $\mathrm{CD} 4+\mathrm{T}$ cell differentiation towards a Th2 profile. Together, these observations suggest that CD8+ $\mathrm{T}$ cells may favor the survival of resting CD4+ T cells carrying integrated HIV provirus.

\section{Promising LRA and LRA Combinations}

In addition to the LRA mentioned above, there are several new agents or combinations of agents that we are likely to hear more about in the coming years, based on promising in vitro work. These include additional TLR stimulators, including a dual TLR-2 and -7 agonist [76], and others targeting TLR-8 [77], or TLR-1/2 that may act directly on CD4+ T cells [78]. Fimepinostat, that inhibits both PI3K and HDAC, was shown to increase levels of US HIV-1 RNA in CD4+ T cells from donors on long-term ART without causing activation of central or effector memory CD4+ T cells [79]. In another approach to specifically target intracellular signaling pathways in CD4+ T cells, Bosque and colleagues identified benzotriazoles as inhibitors of the SUMOlyation (and thus inactivation) of STAT5 [80]. Treatment of CD4+ T cells from ART-suppressed donors with benzotriazole + IL-2 resulted in p24 release without an increase in cellular activation or proliferation. Interestingly, the effect required IL-2, a member of the $\gamma$-chain cytokine family that also includes IL-15 and IL-7 which also activate STAT5 [81]. Several trials of IL-15 (as the superagonist N-803) alone or in combination with other agents are planned or underway [82]. IL-7 also has also been shown to induce p24 expression from thymocytes and splenocytes from HIV-1 infected SCID-hu mice stimulated ex vivo in the presence of ART [83]. As discussed above, IL-2 was toxic when given to HIV-infected trial participants, and while the in vitro results described in this section are encouraging, all of these approaches will need to be validated for safety and efficacy in pre-clinical nonhuman primate models or clinical trials. 
Relatively new pharmacologic agents termed senotherapeutics function to target cells in their senescence, a state characterized by pro-inflammatory cell cycle arrest. Such compounds include senolytics which lead to elimination of senescent cells and senomorphics which inhibit or suppress the senescence-associated secretory phenotype (SASP) (that encompasses the collective secreted factors promoting inflammation during senescence) [84]. It has been previously shown that persistent immune activation due to chronic HIV infection may hasten immunosenescence, or immune aging [85]. This led to the investigation of select senotherapeutic compounds as potential HIV curative agents targeting latently infected CD4+ T cells through mechanisms including latency reversal, targeted apoptosis, and anti-proliferation [86]. The senomorphic mechanistic target of rapamycin (mTOR) inhibitor sirolimus (or rapamycin) has been studied for its anti-proliferative properties in latent HIV infection. With regard to latency reversal, Martin and colleagues showed that resting CD4+ T cells from ART-suppressed individuals showed equivalent induction of HIV mRNA upon $\alpha \mathrm{CD} 3 / \alpha \mathrm{CD} 28$ stimulation in the presence or absence of rapamycin and, importantly, rapamycin was associated with significantly reduced production of pro-inflammatory cytokines [87]. In the same study, coupling of rapamycin with the PKC agonist bryostatin- 1 or HDACi romidepsin did not significantly affect HIV mRNA production but did inhibit stimulationinduced cytokine release. A similar interaction has been reported between the PKC agonist ingenol B and the Janus kinase (JAK) inhibitor ruxolitinb [88]. Overall, this suggests that use of global $\mathrm{T}$ cell activators in the presence of mTOR or JAK inhibitors may reduce toxicity and ultimately open particular LRA 1.0 candidates for reconsideration.

\section{LRA 2.0 in Shock and Kill Strategies}

A stable viral reservoir size following LRA administration suggests an absence of or insufficient clearance of infected cells by virus-induced cytopathic effect or unboosted immune effector cells. With better understanding of latency reversal and now several approaches available that induce readily measurable onART viremia, several groups are thus exploring in vivo combination LRA 2.0 plus clearance agents in nonhuman primate models. We recently used bispecific $\mathrm{HIVxCD} 3$ retargeting molecules in combination with the SMACm AZD5582 in a model of ART-suppressed SHIV-infected RM [89], but unexpectedly did not observe latency reversal that we attributed to a small reservoir size in this model. We are currently using the model of ARTsuppressed RM infected with SIV to assess the impact on the viral reservoir of a combination of a cocktail of SIV-specific neutralizing and non-neutralizing monoclonal antibodies given with either AZD5582 or N-803 plus the CD8 $\alpha$-depleting antibody MT-807R1. Additional approaches that may prove successful include "shock and suicide" in which reactivated cells are specifically induced to die with inhibitors of prosurvival proteins or "surge and purge" in which both the LRA/immune stimulator and clearance agent are given coincident with ART initiation early in infection to restrict reservoir establishment.

\section{Remaining Questions About Latency Reversal as a Component of an HIV Cure Strategy}

With the recent successes in achieving robust latency reversal in multiple animal models as described above, our attention is now focused on capitalizing on these LRA in combination shock and kill experiments that include a clearance arm to reduce or eliminate persistent reservoirs. However, several outstanding questions remain regarding the effect and consequences of latency reversal with SMACm and/or N803 + CD8 lymphocyte depletion. First, what are the specific cellular and anatomic origins of reactivated virus that lead to on-ART viremia; second, might these immune stimulating approaches have the unintended consequence of expanding the reservoir through promotion of cellular proliferation and clonal expansion; third, what depth and extent of latency reversal is needed to provide sufficient fodder for clearance agents to measurably change the reservoir size; and fourth, is there a differential susceptibility of CD4+ T cell subsets with reactivated virus to diverse elimination strategies? The answer to these questions will come from future studies that not only assess the efficacy of LRA to reactivate latent virus but also specifically address these more mechanistic considerations. Prioritizing experiments designed to dig deeper into the consequences of latency reversal and using the information gained to optimize approaches to eliminate reactivated cells is likely to significantly advance the HIV cure field.

Acknowledgments We gratefully thank Deanna Kulpa for her critical reading of the manuscript.

Funding A.C. acknowledges funding from the National Institutes of Health grants R01 AI133706, R01 AI125064, UM1 AI124436, and UM1 AI126619.

\section{Compliance with Ethical Standards}

Conflict of Interest The authors declare that they have no conflict of interest.

Open Access This article is licensed under a Creative Commons Attribution 4.0 International License, which permits use, sharing, adaptation, distribution and reproduction in any medium or format, as long as you give appropriate credit to the original author(s) and the source, provide a link to the Creative Commons licence, and indicate if changes were made. The images or other third party material in this article are included in the article's Creative Commons licence, unless indicated otherwise in a credit line to the material. If material is not included in the article's 
Creative Commons licence and your intended use is not permitted by statutory regulation or exceeds the permitted use, you will need to obtain permission directly from the copyright holder. To view a copy of this licence, visit http://creativecommons.org/licenses/by/4.0/.

\section{References}

Papers of particular interest, published recently, have been highlighted as:

- Of importance

-• Of major importance

1. Chun TW, Engel D, Mizell SB, Hallahan CW, Fischette M, Park S, et al. Effect of interleukin-2 on the pool of latently infected, resting CD4+ T cells in HIV-1-infected patients receiving highly active anti-retroviral therapy. Nat Med. 1999;5:651-5.

2. Dybul M, Hidalgo B, Chun TW, Belson M, Migueles SA, Justement JS, et al. Pilot study of the effects of intermittent interleukin-2 on human immunodeficiency virus (HIV)-specific immune responses in patients treated during recently acquired HIV infection. J Infect Dis. 2002;185:61-8.

3. Prins JM, Jurriaans S, van Praag RM, Blaak H, van Rij R, Schellekens PT, et al. Immuno-activation with anti-CD3 and recombinant human IL-2 in HIV-1-infected patients on potent antiretroviral therapy. AIDS. 1999;13:2405-10.

4. van Praag RM, Prins JM, Roos MT, Schellekens PT, Ten Berge IJ, Yong SL, et al. OKT3 and IL-2 treatment for purging of the latent HIV-1 reservoir in vivo results in selective long-lasting CD4+ T cell depletion. J Clin Immunol. 2001;21:218-26.

5. Lehrman G, Hogue IB, Palmer S, Jennings C, Spina CA, Wiegand A, et al. Depletion of latent HIV-1 infection in vivo: a proof-ofconcept study. Lancet. 2005;366:549-55.

6. Siliciano JD, Lai J, Callender M, Pitt E, Zhang H, Margolick JB, et al. Stability of the latent reservoir for HIV-1 in patients receiving valproic acid. J Infect Dis. 2007;195:833-6.

7. Archin NM, Liberty AL, Kashuba AD, Choudhary SK, Kuruc JD, Crooks AM, et al. Administration of vorinostat disrupts HIV-1 latency in patients on antiretroviral therapy. Nature. 2012;487: 482-5.

8. Archin NM, Bateson R, Tripathy MK, Crooks AM, Yang KH, Dahl NP, et al. HIV-1 expression within resting CD4+ T cells after multiple doses of vorinostat. J Infect Dis. 2014;210:728-35.

9. Elliott JH, Wightman F, Solomon A, Ghneim K, Ahlers J, Cameron $\mathrm{MJ}$, et al. Activation of HIV transcription with short-course vorinostat in HIV-infected patients on suppressive antiretroviral therapy. PLoS Pathog. 2014;10:e1004473.

10. Li H, Wang S, Kong R, Ding W, Lee FH, Parker Z, et al. Envelope residue 375 substitutions in simian-human immunodeficiency viruses enhance $\mathrm{CD} 4$ binding and replication in rhesus macaques. Proc Natl Acad Sci U S A. 2016;113:E3413-22.

11. Palmer S, Wiegand AP, Maldarelli F, Bazmi H, Mican JM, Polis M, et al. New real-time reverse transcriptase-initiated PCR assay with single-copy sensitivity for human immunodeficiency virus type 1 RNA in plasma. J Clin Microbiol. 2003;41:4531-6.

12. Swanstrom AE, Gorelick RJ, Wu G, Howell B, Vijayagopalan A, Shoemaker R, et al. Ultrasensitive immunoassay for simian immunodeficiency virus p27(CA). AIDS Res Hum Retrovir. 2018;34: 993-1001.

13. Cillo AR, Sobolewski MD, Bosch RJ, Fyne E, Piatak M Jr, Coffin $\mathrm{JM}$, et al. Quantification of HIV-1 latency reversal in resting CD4+
$\mathrm{T}$ cells from patients on suppressive antiretroviral therapy. Proc Natl Acad Sci U S A. 2014;111:7078-83.

14. Pasternak AO, de Bruin M, Jurriaans S, Bakker M, Berkhout B, Prins JM, et al. Modest nonadherence to antiretroviral therapy promotes residual HIV-1 replication in the absence of virological rebound in plasma. J Infect Dis. 2012;206:1443-52.

15. Yukl SA, Kaiser P, Kim P, Telwatte S, Joshi SK, Vu M, et al. HIV latency in isolated patient CD4(+) T cells may be due to blocks in HIV transcriptional elongation, completion, and splicing. Sci Transl Med. 2018;10.

16. Bullen CK, Laird GM, Durand CM, Siliciano JD, Siliciano RF. New ex vivo approaches distinguish effective and ineffective single agents for reversing HIV-1 latency in vivo. Nat Med. 2014;20:425-9.

17. Bertoldi A, D'Urbano V, Bon I, Verbon A, Rokx C, Boucher C, et al. Development of C-TILDA: a modified TILDA method for reservoir quantification in long term treated patients infected with subtype C HIV-1. J Virol Methods. 2020;276:113778.

18. Frank I, Acharya A, Routhu NK, Aravantinou M, Harper JL, Maldonado S, et al. A tat/rev induced limiting dilution assay to measure viral reservoirs in non-human primate models of HIV infection. Sci Rep. 2019;9:12078.

19. Procopio FA, Fromentin R, Kulpa DA, Brehm JH, Bebin AG, Strain MC, et al. A novel assay to measure the magnitude of the inducible viral reservoir in $\mathrm{HIV}$-infected individuals. EBioMedicine. 2015;2:874-83.

20. Deleage C, Chan CN, Busman-Sahay K, Estes JD. Next-generation in situ hybridization approaches to define and quantify HIV and SIV reservoirs in tissue microenvironments. Retrovirology. 2018;15:4.

21. Zhang W, Svensson Akusjarvi S, Sonnerborg A, Neogi U. Characterization of inducible transcription and translationcompetent HIV-1 using the RNAscope ISH technology at a single-cell resolution. Front Microbiol. 2018;9:2358.

22. Baxter AE, Niessl J, Fromentin R, Richard J, Porichis F, Massanella $\mathrm{M}$, et al. Multiparametric characterization of rare HIV-infected cells using an RNA-flow FISH technique. Nat Protoc. 2017;12:2029-49.

23. Martrus G, Niehrs A, Cornelis R, Rechtien A, Garcia-Beltran W, Lutgehetmann M, et al. Kinetics of HIV-1 latency reversal quantified on the single-cell level using a novel flow-based technique. J Virol. 2016;90:9018-28.

24. Rasmussen TA, Tolstrup M, Brinkmann CR, Olesen R, Erikstrup $\mathrm{C}$, Solomon A, et al. Panobinostat, a histone deacetylase inhibitor, for latent-virus reactivation in HIV-infected patients on suppressive antiretroviral therapy: a phase $1 / 2$, single group, clinical trial. Lancet HIV. 2014;1:e13-21.

25. Sogaard OS, Graversen ME, Leth S, Olesen R, Brinkmann CR, Nissen SK, et al. The depsipeptide romidepsin reverses HIV-1 latency in vivo. PLoS Pathog. 2015;11:e1005142.

26. Bouchat S, Gatot JS, Kabeya K, Cardona C, Colin L, Herbein G, et al. Histone methyltransferase inhibitors induce HIV-1 recovery in resting CD4(+) T cells from HIV-1-infected HAART-treated patients. AIDS. 2012;26:1473-82.

27. Li Z, Guo J, Wu Y, Zhou Q. The BET bromodomain inhibitor JQ1 activates HIV latency through antagonizing Brd4 inhibition of Tattransactivation. Nucleic Acids Res. 2013;41:277-87.

28. Lu P, Shen Y, Yang H, Wang Y, Jiang Z, Yang X, et al. BET inhibitors RVX-208 and PFI-1 reactivate HIV-1 from latency. Sci Rep. 2017;7:16646.

29. Lim SY, Osuna CE, Hraber PT, Hesselgesser J, Gerold JM, Barnes TL, et al. TLR7 agonists induce transient viremia and reduce the viral reservoir in SIV-infected rhesus macaques on antiretroviral therapy. Sci Transl Med. 2018;10 This work showed that TLR7 agonist (GS-986 and GS-9620) treatment induced upregulation of antiviral genes, transient on-ART viremia, and reduction in the viral reservoir size in SIV-infected macaques. 
30. Del Prete GQ, Alvord WG, Li Y, Deleage C, Nag M, Oswald K, et al. TLR7 agonist administration to SIV-infected macaques receiving early initiated cART does not induce plasma viremia. JCI Insight. 2019;4 This study demonstrated that repeat dosing of the TLR7 agonist GS-9620 in SIV-infected macaques receiving early, long-term ART did not result in measurable increases in on-ART viremia suggesting that latency reversal with TLR-7 agonism could depend on the timing and duration of ART.

31. Bekerman E, Hesselgesser J, Carr B, Nagel M, Hung M, Wang A, et al. PD-1 blockade and TLR7 activation lack therapeutic benefit in chronic simian immunodeficiency virus-infected macaques on antiretroviral therapy. Antimicrob Agents Chemother. 2019;63 Here, SIV-infected macaques initiated on ART during chronic infection and treated with the TLR7 agonist GS-9620 (or GS-9620 plus PD-1 blockade) did not have evidence of on-ART viremia nor a difference in viral reservoir size and viral rebound kinetics after ART interruption compared to control macaques.

32. Borducchi EN, Cabral C, Stephenson KE, Liu J, Abbink P, Ng'ang'a D, et al. Ad26/MVA therapeutic vaccination with TLR7 stimulation in SIV-infected rhesus monkeys. Nature. 2016;540:284-7 In the first report of TLR-7 stimulation with GS-986 in SIV-infected, ART-suppressed macaques, there were no increases seen in on-ART viremia in the GS-986 only group. TLR-7 stimulation in combination with therapeutic vaccination did result in longer time to viral rebound and lower viral set point after ART interruption.

33. Borducchi EN, Liu J, Nkolola JP, Cadena AM, Yu WH, Fischinger $\mathrm{S}$, et al. Antibody and TLR7 agonist delay viral rebound in SHIVinfected monkeys. Nature. 2018;563:360-4.

34. Bricker K, Obregon-Perko V, Uddin F, Williams B, Uffmna EA, Garrido C, et al. (in press) Therapeutic vaccination of SIV-infected, ART-treated infant rhesus macaques using Ad48/MVA in combination with TLR-7 stimulation. PLoS Pathog. 2020;16:e1008954.

35. Riddler SA, Para M, Benson CA, Mills A, Ramgopal M, DeJesus E, et al. Vesatolimod, a toll-like receptor 7 agonist, induces immune activation in virally suppressed adults with HIV-1. Clin Infect Dis. 2020. https://doi.org/10.1093/cid/ciaa1534.

36. Vibholm L, Schleimann MH, Hojen JF, Benfield T, Offersen R, Rasmussen K, et al. Short-course toll-like receptor 9 agonist treatment impacts innate immunity and plasma viremia in individuals with human immunodeficiency virus infection. Clin Infect Dis. 2017;64:1686-95.

37. Vibholm LK, Konrad CV, Schleimann MH, Frattari G, Winckelmann A, Klastrup V, et al. Effects of 24-week Toll-like receptor 9 agonist treatment in HIV type 1+ individuals. AIDS. 2019;33:1315-25.

38. Winckelmann AA, Munk-Petersen LV, Rasmussen TA, Melchjorsen J, Hjelholt TJ, Montefiori D, et al. Administration of a Toll-like receptor 9 agonist decreases the proviral reservoir in virologically suppressed HIV-infected patients. PLoS One. 2013;8:e62074.

39. Saxena M, Sabado RL, La Mar M, Mohri H, Salazar AM, Dong H, et al. Poly-ICLC, a TLR3 agonist, induces transient innate immune responses in patients with treated HIV-infection: a randomized double-blinded placebo controlled trial. Front Immunol. 2019;10: 725.

40. Li P, Kaiser P, Lampiris HW, Kim P, Yukl SA, Havlir DV, et al. Stimulating the RIG-I pathway to kill cells in the latent HIV reservoir following viral reactivation. Nat Med. 2016;22:807-11.

41. Yamamoto T, Kanuma T, Takahama S, Okamura T, Moriishi E, Ishii KJ, et al. STING agonists activate latently infected cells and enhance SIV-specific responses ex vivo in naturally SIV controlled cynomolgus macaques. Sci Rep. 2019;9:5917.

42. Palermo E, Acchioni C, Di Carlo D, Zevini A, Muscolini M, Ferrari $\mathrm{M}$, et al. Activation of latent HIV-1 T cell reservoirs with a combination of innate immune and epigenetic regulators. J Virol. 2019;93.

43. Chomont N, El-Far M, Ancuta P, Trautmann L, Procopio FA, Yassine-Diab B, et al. HIV reservoir size and persistence are driven by T cell survival and homeostatic proliferation. Nat Med. 2009;15: 893-900.

44. Evans VA, van der Sluis RM, Solomon A, Dantanarayana A, McNeil C, Garsia R, et al. Programmed cell death-1 contributes to the establishment and maintenance of HIV-1 latency. AIDS. 2018;32:1491-7.

45. Fromentin R, Bakeman W, Lawani MB, Khoury G, Hartogensis W, DaFonseca $\mathrm{S}$, et al. CD4+ T cells expressing PD-1, TIGIT and LAG-3 contribute to HIV persistence during ART. PLoS Pathog. 2016;12:e1005761.

46. McGary CS, Deleage C, Harper J, Micci L, Ribeiro SP, Paganini S, et al. CTLA-4(+)PD-1(-) memory CD4(+) T cells critically contribute to viral persistence in antiretroviral therapy-suppressed, SIVInfected Rhesus Macaques. Immunity. 2017;47(776-788):e5.

47. Pardons M, Fromentin R, Pagliuzza A, Routy JP, Chomont N. Latency-reversing agents induce differential responses in distinct memory CD4 $\mathrm{T}$ cell subsets in individuals on antiretroviral therapy. Cell Rep. 2019;29(2783-2795):e5.

48. Chen H, Moussa M, Catalfamo M. The role of immunomodulatory receptors in the pathogenesis of HIV infection: a therapeutic opportunity for HIV cure? Front Immunol. 2020;11:1223.

49. Wightman F, Solomon A, Kumar SS, Urriola N, Gallagher K, Hiener B, et al. Effect of ipilimumab on the HIV reservoir in an HIV-infected individual with metastatic melanoma. AIDS. 2015;29:504-6.

50. Guihot A, Marcelin AG, Massiani MA, Samri A, Soulie C, Autran B, et al. Drastic decrease of the HIV reservoir in a patient treated with nivolumab for lung cancer. Ann Oncol. 2018;29:517-8.

51. Le Garff G, Samri A, Lambert-Niclot S, Even S, Lavole A, Cadranel J, et al. Transient HIV-specific T cells increase and inflammation in an HIV-infected patient treated with nivolumab. AIDS. 2017;31:1048-51.

52. Scully EP, Rutishauser RL, Simoneau CR, Delagreverie H, Euler Z, Thanh $\mathrm{C}$, et al. Inconsistent HIV reservoir dynamics and immune responses following anti-PD-1 therapy in cancer patients with HIV infection. Ann Oncol. 2018;29:2141-2.

53.• Harper J, Gordon S, Chan CN, Wang H, Lindemuth E, Galardi C, et al. CTLA-4 and PD-1 dual blockade induces SIV reactivation without control of rebound after antiretroviral therapy interruption. Nat Med. 2020;26:519-28 This study demonstrated that in SIVinfected long-term ART-treated macaques, CTLA-4/PD-1 blockade was more effective in inducing latency reversal and reducing integrated virus in CD4+ $T$ cells compared to PD-1 monotherapy alone, but had limited impact on viral rebound after ART interruption.

54. Connolly MA, Gayer M, Ryan MJ, Salama P, Spiegel P, Heymann DL. Communicable diseases in complex emergencies: impact and challenges. Lancet. 2004;364:1974-83.

55. Gay C, Hardy D. Safety and immunotherapeutic activity of an antiPD-1 antibody (cemiplimab) in HIV-1-diagnosed participants on suppressive cART: a phase I/II, double-blind, placebo-controlled, ascending multiple dose study, p. In (ed),

56. Gilmore TD. Introduction to NF-kappaB: players, pathways, perspectives. Oncogene. 2006;25:6680-4.

57. Sun SC. The non-canonical NF-kappaB pathway in immunity and inflammation. Nat Rev Immunol. 2017;17:545-58.

58. Sun SC. Non-canonical NF-kappaB signaling pathway. Cell Res. 2011;21:71-85.

59. Liao G, Zhang M, Harhaj EW, Sun SC. Regulation of the NFkappaB-inducing kinase by tumor necrosis factor receptorassociated factor 3-induced degradation. J Biol Chem. 2004;279: 26243-50. 
60. Gutierrez C, Serrano-Villar S, Madrid-Elena N, Perez-Elias MJ, Martin ME, Barbas C, et al. Bryostatin-1 for latent virus reactivation in HIV-infected patients on antiretroviral therapy. AIDS. 2016;30:1385-92.

61. Elliott JH, McMahon JH, Chang CC, Lee SA, Hartogensis W, Bumpus N, et al. Short-term administration of disulfiram for reversal of latent HIV infection: a phase 2 dose-escalation study. Lancet HIV. 2015;2:e520-9.

62. Spivak AM, Andrade A, Eisele E, Hoh R, Bacchetti P, Bumpus $\mathrm{NN}$, et al. A pilot study assessing the safety and latency-reversing activity of disulfiram in HIV-1-infected adults on antiretroviral therapy. Clin Infect Dis. 2014;58:883-90.

63.• Pache L, Dutra MS, Spivak AM, Marlett JM, Murry JP, Hwang Y, et al. BIRC2/cIAP1 is a negative regulator of HIV-1 transcription and can be targeted by Smac mimetics to promote reversal of viral latency. Cell Host Microbe. 2015;18:345-53 This report first described the potential of SMACm to activate the ncNF- $\mathrm{KB}$ pathway to reverse latency in several in vitro and ex vivo systems.

64. Bobardt M, Kuo J, Chatterji U, Chanda S, Little SJ, Wiedemann N, et al. The inhibitor apoptosis protein antagonist Debio 1143 is an attractive HIV-1 latency reversal candidate. PLoS One. 2019;14: e0211746.

65.• Nixon CC, Mavigner M, Sampey GC, Brooks AD, Spagnuolo RA, Irlbeck DM, et al. Systemic HIV and SIV latency reversal via noncanonical NF-kappaB signalling in vivo. Nature. 2020. https://doi. org/10.1038/s41586-020-1951-3 This study demonstrated, in two complementary animal models, that in vivo treatment with the SMACm AZD5582 induced significant latency reversal measured by increases in HIV/SIV RNA in plasma and in resting CD4+ $T$ cells with minimal side effects.

66. Pache L, Marsden MD, Teriete P, Portillo AJ, Heimann D, Kim JT, et al. Pharmacological activation of non-canonical NF- $\mathrm{KB}$ signaling activates latent HIV-1 reservoirs in vivo. Cell Reports Medicine. 2020;1:100037.

67. Campbell GR, Bruckman RS, Chu YL, Trout RN, Spector SA. SMAC Mimetics induce autophagy-dependent apoptosis of HIV1 -infected resting memory CD4+ T cells. Cell Host Microbe. 2018;24(689-702):e7.

68. Campbell GR, To RK, Zhang G, Spector SA. SMAC mimetics induce autophagy-dependent apoptosis of HIV-1-infected macrophages. Cell Death Dis. 2020;11:590.

69. Walker CM, Moody DJ, Stites DP, Levy JA. CD8+ lymphocytes can control HIV infection in vitro by suppressing virus replication. Science. 1986;234:1563-6.

70. McBrien JB, Wong AKH, White E, Carnathan DG, Lee JH, Safrit JT, et al. Combination of CD8beta depletion and interleukin-15 superagonist $\mathrm{N}-803$ induces virus reactivation in simian-human immunodeficiency virus-infected, long-term ART-treated rhesus macaques. J Virol. 2020;94.

71. Cartwright EK, Spicer L, Smith SA, Lee D, Fast R, Paganini S, et al. CD8(+) lymphocytes are required for maintaining viral suppression in SIV-infected macaques treated with short-term antiretroviral therapy. Immunity. 2016;45:656-68.

72.• McBrien JB, Mavigner M, Franchitti L, Smith SA, White E, Tharp $\mathrm{GK}$, et al. Robust and persistent reactivation of SIV and HIV by $\mathrm{N}-803$ and depletion of CD8(+) cells. Nature. 2020. https://doi.org/ 10.1038/s41586-020-1946-0 Using multiple models, this work demonstrated robust latency reversal evidenced by prolonged on-ART viremia with the IL-15 superagonist $\mathrm{N}-803$ in combination with depletion of CD8 $\alpha$ expresing lymphocytes. This LRA combination induced significantly greater and more persistent viremia than either agent alone further highlighting the role of CD8+ cells in suppressing virus reactivation.

73. Jones RB, Mueller S, O'Connor R, Rimpel K, Sloan DD, Karel D, et al. A subset of latency-reversing agents expose HIV-infected resting CD4+ T-cells to recognition by cytotoxic T-lymphocytes. PLoS Pathog. 2016;12:e1005545.

74. Mavigner M, Liao L, Brooks A, Ke R, Mattingly C, Schoof N, Liang S, Vanderford T, Paiardini M, Kulpa D, Lifson J, Dunham R, Margolis D, Perelson A, Silvestri G, Chahroudi A. 2020. CD8 lymphocyte depletion enhances the latency reversal activity of the SMAC mimetic AZD5582 in ART-suppressed SIV-infected rhesus macaques JVI [under review].

75. Zanoni M, Palesch D, Pinacchio C, Statzu M, Tharp GK, Paiardini $\mathrm{M}$, et al. Innate, non-cytolytic CD8+ T cell-mediated suppression of HIV replication by MHC-independent inhibition of virus transcription. PLoS Pathog. 2020;16:e1008821.

76. Macedo AB, Novis CL, De Assis CM, Sorensen ES, Moszczynski $\mathrm{P}$, Huang SH, et al. Dual TLR2 and TLR7 agonists as HIV latencyreversing agents. JCI Insight. 2018;3.

77. Schlaepfer E, Speck RF. TLR8 activates HIV from latently infected cells of myeloid-monocytic origin directly via the MAPK pathway and from latently infected CD4+ T cells indirectly via TNF-alpha. J Immunol. 2011;186:4314-24.

78. Novis CL, Archin NM, Buzon MJ, Verdin E, Round JL, Lichterfeld $\mathrm{M}$, et al. Reactivation of latent HIV-1 in central memory CD4(+) T cells through TLR-1/2 stimulation. Retrovirology. 2013;10:119.

79. Gunst JD, Kjaer K, Olesen R, Rasmussen TA, Ostergaard L, Denton PW, et al. Fimepinostat, a novel dual inhibitor of HDAC and PI3K, effectively reverses HIV-1 latency ex vivo without $\mathrm{T}$ cell activation. J Virus Erad. 2019;5:133-7.

80. Bosque A, Nilson KA, Macedo AB, Spivak AM, Archin NM, Van Wagoner RM, et al. Benzotriazoles reactivate latent HIV-1 through inactivation of STAT5 SUMOylation. Cell Rep. 2017;18:1324-34.

81. Chahroudi A, Silvestri G. Interleukin-7 in HIV pathogenesis and therapy. Eur Cytokine Netw. 2010;21:202-7.

82. Anonymous. ClinicalTrials.gov. https://clinicaltrials.gov/ct2/ results ? cond $=\&$ term $=\mathrm{N} 803 \&$ cntry $=\&$ state $=\&$ city $=\&$ dist $=$. Accessed.

83. Scripture-Adams DD, Brooks DG, Korin YD, Zack JA. Interleukin-7 induces expression of latent human immunodeficiency virus type 1 with minimal effects on T-cell phenotype. J Virol. 2002; 76:13077-82.

84. Childs BG, Gluscevic M, Baker DJ, Laberge RM, Marquess D, Dananberg J, et al. Senescent cells: an emerging target for diseases of ageing. Nat Rev Drug Discov. 2017;16:718-35.

85. Nelson JA, Krishnamurthy J, Menezes P, Liu Y, Hudgens MG, Sharpless NE, et al. Expression of p16(INK4a) as a biomarker of $\mathrm{T}$-cell aging in HIV-infected patients prior to and during antiretroviral therapy. Aging Cell. 2012;11:916-8.

86. Szaniawski MA, Spivak AM. Senotherapeutics and HIV-1 persistence. Curr HIV/AIDS Rep. 2020;17:219-25.

87. Martin AR, Pollack RA, Capoferri A, Ambinder RF, Durand CM, Siliciano RF. Rapamycin-mediated mTOR inhibition uncouples HIV-1 latency reversal from cytokine-associated toxicity. J Clin Invest. 2017;127:651-6.

88. Spivak AM, Larragoite ET, Coletti ML, Macedo AB, Martins LJ, Bosque A, et al. Janus kinase inhibition suppresses PKC-induced cytokine release without affecting HIV-1 latency reversal ex vivo. Retrovirology. 2016;13:88.

89. Dashti A, Waller C, Mavigner M, Schoof N, Bar KJ, Shaw GM, et al. SMAC mimetic plus triple combination bispecific HIVxCD3 DART(R) molecules in SHIV.C.CH505-infected, ART-suppressed rhesus macaques. J Virol. 2020. https://doi.org/10.1128/JVI.00793-20.

Publisher's Note Springer Nature remains neutral with regard to jurisdictional claims in published maps and institutional affiliations. 